\title{
Limit-analysis of a circular cylinder obeying the Green plasticity criterion and loaded in combined tension and torsion
}

\author{
Nadjime Pindra ${ }^{1}$, Jean-Baptiste Leblond ${ }^{2}{ }^{*}$, Djimédo Kondo ${ }^{2}$ \\ ${ }^{1}$ Université de Lomé, Faculté des Sciences, Département de Mathématiques, Lomé, Togo \\ ${ }^{2}$ Sorbonne Université, Faculté des Sciences (formerly Université Pierre et Marie Curie), \\ CNRS, UMR 7190, Institut Jean Le Rond d'Alembert, Tour 65-55, 4 place Jussieu, 75252 \\ Paris Cedex 05, France
}

\begin{abstract}
This short paper presents the limit-analysis of a cylinder with circular basis, made of an idealplastic material obeying Green's yield criterion and subjected to combined tension and torsion. The exact solution of the problem is provided in the form of a statically and plastically admissible stress field and a kinematically admissible velocity field, associated via the normality rule. The overall yield locus, that is the set of pairs [tension force, torsion torque] for which unrestrained plastic flow occurs, is expressed first in parametric form, then explicitly upon elimination of the parameter involved. The explicit expression of this yield locus also entails that of the overall flow rule via the overall normality property. The impact of these results is two-fold. First, they provide a fresh example of a solution to a limit-analysis problem exceptionally combining three generally mutually exclusive features: be non-trivial, exact and explicit. Second, they provide a way of using simple experiments of combined tension and torsion of cylinders to determine the parameter characterizing the influence of the mean stress in Green's criterion.

Keywords: Circular cylinder; combined tension and torsion; Green material; limit-analysis; parametric yield locus; explicit yield locus
\end{abstract}

\section{Introduction}

The problem of a circular cylinder made of some elastic-ideal-plastic von Mises material and subjected to combined tension and torsion is only slightly less popular than the corresponding one for a thin tube, and has been treated several times in classical papers and textbooks (Hill, 1950; Prager and Hodge, 1951; Gaydon, 1952; Drucker, 1967; Avitzur and Pan, 1985; Chakrabarty, 1987; de Buhan, 2007; Lubliner, 2008).

$\bar{*}$ Corresponding author. 
The elastic-plastic solution contains in particular, in the limit of very large values of the torsion angle and/or the axial strain, the values of the limit-loads promoting unrestrained plastic flow of the structure, for arbitrary values of the ratio of the torsion torque to the tension force. (These limit-loads coincide with those which would induce plastic flow in the limiting case of a rigid-ideal-plastic behaviour, that is in the absence of elasticity). ${ }^{1}$ The study of limit-loads is the object of limit-analysis, which provides direct methods - the static and kinematic approaches - to determine them without solving the entire evolution problem for an elastic-plastic structure; see, on this topic, the seminal works of Hill (1951) and Prager and Hodge (1951), and the more modern presentations of Salençon (1983) and Save et al. (1997)).

Previous authors provided parametric expressions of the tension force and torsion torque at a limit-load, in terms of the ratio of the torsional angular rate to the axial strain rate. Lubliner (2008) managed to eliminate the parameter involved in these parametric expressions and obtained in that way the equation of the overall yield locus - that is the set of pairs [tension force, torsion torque] defining the limit-loads - in a simple and fully explicit form.

The aim of this paper is to extend Lubliner (2008)'s solution to materials obeying Green (1972)'s yield criterion and the associated flow rule. Green (1972)'s criterion heuristically extends that of von Mises by including an influence of the first invariant of the stress in quadratic form, and represents the simplest envisageable criterion for porous solids with arbitrary porosity. It is applicable to highly porous metals such as foams, and also some very porous clays or chalks, see for instance Shao and Henry (1991) and Nadah et al. (2013). (For low porosities it is surpassed by Gurson (1977)'s famous criterion based on detailed micromechanical analysis and homogenization). It has been put to use by Kuhn and Downey (1971), Shima and Oyane (1976), Corapcioglu and Uz (1978), Oyane et al. (1989), Shen et al. (2012) and Fritzen et al. (2013), among others.

Thanks to the simplicity of the geometrical configuration, we shall exhibit both a statically and plastically admissible stress field and a kinematically admissible velocity field associated via the flow rule, which will together represent the exact solution of the limitanalysis problem by virtue of Salençon (1983)'s "association theorem". (Another basically equivalent, but less rigorous point of view, will be that we shall construct the exact solution for an ideal-plastic material in the absence of elasticity). The explicit equation of the overall yield locus will be found. The benefits of the solution found will be two-fold:

- It will enrich the literature with a fresh example of a solution to some limit-analysis problem which is simultaneously non-trivial, exact and explicit - three features seldom found to be compatible.

- More importantly, it will provide a means of determining the parameter characterizing the effect of the mean stress in Green's criterion, through experiments of combined tension and torsion of cylindrical specimens.

$\overline{1}$ Drucker et al. (1952) have shown that when a structure made of some elastic-ideal-plastic material reaches a limit-load, the elastic strain rate is zero at all points of this structure, which means that it behaves as if its constitutive material were rigid-ideal-plastic. 
The paper is organized as follows:

- Section 2 is devoted to notations and preliminaries.

- Section 3 presents the exact solution of the limit-analysis problem, in the form of explicit expressions of the stress and velocity fields.

- Section 4 deduces from there parametric equations of the overall yield locus, using the ratio of the torsional angular rate to the axial strain rate as a parameter.

- Section 5 explains how to eliminate this parameter, thus leading to an explicit, analytic "overall yield function" for the convex set of sustainable loads.

- Section 6 is devoted to the study of the overall flow rule, and in particular the verification of the property of overall normality predicted by the theory of limit-analysis.

- Finally Section 7 discusses the comparison with the case of a thin tube, and the practical application of the results derived.

\section{Notations and preliminaries}

We consider a circular cylinder of radius $R$, height $H$, subjected to a combination of a tension force $F$ and a torsion torque $T$, in the absence of body forces (Figure 1). The constitutive material of this cylinder is supposed be ideal-plastic (no strain hardening) and obey Green (1972)'s plasticity criterion (see below) and the plastic flow rule associated to it through the normality property. The problem will be solved within the linearized framework (small displacements, small strains) classically imposed by the theory of limitanalysis.

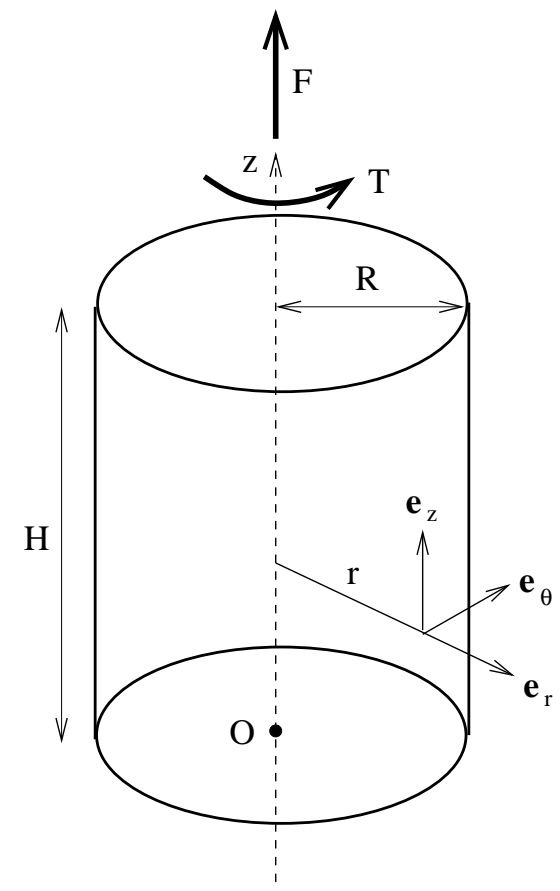

Fig. 1. Circular cylinder subjected to combined tension and torsion

Use is made of cylindrical coordinates $r, \theta, z$ with origin $O$ placed at the center of the lower face of the cylinder; vectors and tensors are expressed through their components in the 
associated local orthonormal basis $\left(\mathbf{e}_{r}, \mathbf{e}_{\theta}, \mathbf{e}_{z}\right)$. Using this basis, the boundary conditions considered read

$$
\begin{cases}\sigma_{z r}=0, \quad v_{\theta}=0, \quad v_{z}=0 & \text { on the lower face } z=0 \\ \sigma_{z r}=0, \quad v_{\theta}=\dot{\phi} r, \quad v_{z}=\dot{\delta} & \text { on the upper face } z=H \\ \sigma_{r r}=0, \quad \sigma_{r \theta}=0, \quad \sigma_{r z}=0 & \text { on the lateral face } r=R\end{cases}
$$

where $\mathbf{v}$ denotes the local velocity, $\boldsymbol{\sigma}$ the local Cauchy stress tensor, $\dot{\delta}$ the extension rate (relative axial velocity of the upper and lower faces) and $\dot{\phi}$ the torsional angular rate (relative rate of rotation of the upper and lower faces about the $O z$ axis).

The solution for the velocity field will be looked for in the following form:

$$
\left\{\begin{array}{l}
v_{r}=a r \\
v_{\theta}=b r z \\
v_{z}=c z
\end{array}\right.
$$

where $a, b$ and $c$ are parameters. (The linearity of $v_{z}$ in $z$ is intuitively clear. For an incompressible material, the expression of $v_{r}$ directly follows, with $a=-c / 2$. For a compressible Green material, the linearity of $v_{r}$ in $r$ becomes far less obvious a priori, but it will turn out that a solution may be found with this assumption).

The solution for the stress field is looked for in the following form: the components $\sigma_{r \theta}$ and $\sigma_{r z}$ of the stress field are assumed to be uniformly zero, and the other components $\sigma_{r r}, \sigma_{\theta \theta}, \sigma_{z z}, \sigma_{\theta z}$ functions of the sole radial coordinate $r$. (The components $\sigma_{r r}$ and $\sigma_{\theta \theta}$ will eventually turn out to also be uniformly zero; some comments on this point will be offered below).

The quantities $b$ and $c$ are related to the kinematic parameters $\dot{\delta}$ and $\dot{\phi}$ through the following relations resulting from the boundary conditions (1):

$$
\dot{\delta}=c H \quad ; \quad \dot{\phi}=b H
$$

The parameter $a$ remains to be determined.

Finally the tension force $F$ and the torsional torque $T$ are related to the components of the stress field through the obvious relations

$$
F=\int_{0}^{R} 2 \pi r \sigma_{z z}(r) d r \quad ; \quad T=\int_{0}^{R} 2 \pi r^{2} \sigma_{\theta z}(r) d r .
$$

\section{Exact solution for the stress and velocity fields}

We look for a solution in which the entire structure is plastic. (In general, there is no reason why a given structure should be entirely plastic when a limit-load is reached; but 
such a property is satisfied in the specific case considered, as will be evident from the fact that the "fully plastic" solution proposed will satisfy all equations of the problem). Thus Green (1972)'s "elliptical” yield criterion

$$
\sigma_{e q}^{2}+\frac{3}{2} \alpha \sigma_{m}^{2}-\sigma_{0}^{2}=0
$$

is assumed to be satisfied everywhere. In this equation

$$
\sigma_{e q} \equiv\left(\frac{3}{2} \mathbf{s}: \mathbf{s}\right)^{1 / 2}
$$

denotes the von Mises equivalent stress ( $\mathbf{s}$ is the stress deviator),

$$
\sigma_{m} \equiv \frac{1}{3} \operatorname{tr} \boldsymbol{\sigma}
$$

the mean stress, and $\alpha$ and $\sigma_{0}$ material parameters, $\alpha$ being dimensionless and $\sigma_{0}$ homogeneous to a stress. Note that the criterion reduces to that of von Mises when the parameter $\alpha$ is zero.

The plastic flow rule associated to the criterion (5) through the normality property reads

$$
\dot{\boldsymbol{\epsilon}}=\eta\left(3 \mathbf{s}+\alpha \sigma_{m} \mathbf{1}\right) \quad, \quad \eta \geq 0
$$

where $\dot{\boldsymbol{\epsilon}}$ denotes the linearized strain rate, $\eta$ the plastic multiplier and $\mathbf{1}$ the unit secondrank tensor.

The first task is to determine the mean stress and the components of the stress deviator in terms of the parameters $a, b, c$ and the plastic multiplier. The flow rule (8) implies, accounting for the expressions (2) of the components of the velocity field, that

$$
\left\{\begin{array}{l}
3 s_{r r}+\alpha \sigma_{m}=\frac{\dot{\epsilon}_{r r}}{\eta}=3 s_{\theta \theta}+\alpha \sigma_{m}=\frac{\dot{\epsilon}_{\theta \theta}}{\eta}=\frac{a}{\eta} \\
3 s_{z z}+\alpha \sigma_{m}=\frac{\dot{\epsilon}_{z z}}{\eta}=\frac{c}{\eta} \\
3 s_{\theta z}=\frac{\dot{\epsilon}_{\theta z}}{\eta}=\frac{b r}{2 \eta}
\end{array}\right.
$$

It is readily seen from the first two equations of system (9), combined with the property $\operatorname{tr} \mathbf{s}=0$, that

$$
3 \alpha \sigma_{m}=\frac{\dot{\epsilon}_{r r}}{\eta}+\frac{\dot{\epsilon}_{\theta \theta}}{\eta}+\frac{\dot{\epsilon}_{z z}}{\eta}=\frac{2 a+c}{\eta} \Rightarrow \sigma_{m}=\frac{2 a+c}{3 \alpha \eta}
$$


The expressions of the components of $\mathbf{s}$ then follow:

$$
\left\{\begin{array}{l}
s_{r r}=s_{\theta \theta}=\frac{a-c}{9 \eta} \\
s_{z z}=\frac{2(c-a)}{9 \eta} \\
s_{\theta z}=\frac{b r}{6 \eta}
\end{array}\right.
$$

We can now determine the value of the plastic multiplier $\eta$ by calculating the squares of the equivalent stress $\left(\sigma_{e q}^{2}=\frac{3}{2}\left(s_{r r}^{2}+s_{\theta \theta}^{2}+s_{z z}^{2}+2 s_{\theta z}^{2}\right)\right)$ and the mean stress, and applying the criterion (5); one thus gets

$$
\eta=\frac{1}{\sigma_{0}} \sqrt{\frac{b^{2} r^{2}}{12}+\frac{(a-c)^{2}}{9}+\frac{(2 a+c)^{2}}{6 \alpha}} .
$$

Combination of equations (10) and (11) then yields the values of the stress components:

$$
\left\{\begin{array}{l}
\sigma_{r r}=s_{r r}+\sigma_{m}=\sigma_{\theta \theta}=s_{\theta \theta}+\sigma_{m}=\frac{1}{9 \alpha \eta}[(6+\alpha) a+(3-\alpha) c] \\
\sigma_{z z}=s_{z z}+\sigma_{m}=\frac{1}{9 \alpha \eta}[2(3-\alpha) a+(3+2 \alpha) c] \\
\sigma_{\theta z}=s_{\theta z}=\frac{b r}{6 \eta}
\end{array}\right.
$$

where $\eta$ is given by equation (12).

We may now determine the value of the parameter $a$ using the general equilibrium equations in cylindrical coordinates:

$$
\left\{\begin{array}{l}
\frac{\partial \sigma_{r r}}{\partial r}+\frac{1}{r} \frac{\partial \sigma_{r \theta}}{\partial \theta}+\frac{\partial \sigma_{r z}}{\partial z}+\frac{\sigma_{r r}-\sigma_{\theta \theta}}{r}=0 \\
\frac{\partial \sigma_{\theta r}}{\partial r}+\frac{1}{r} \frac{\partial \sigma_{\theta \theta}}{\partial \theta}+\frac{\partial \sigma_{\theta z}}{\partial z}+\frac{2 \sigma_{r \theta}}{r}=0 \\
\frac{\partial \sigma_{z r}}{\partial r}+\frac{1}{r} \frac{\partial \sigma_{z \theta}}{\partial \theta}+\frac{\partial \sigma_{z z}}{\partial z}+\frac{\sigma_{r z}}{r}=0
\end{array}\right.
$$

Since $\sigma_{r r}-\sigma_{\theta \theta}=0$ by the first equation of system (13), these equations reduce to $d \sigma_{r r} / d r=$ 0 , which implies that $\sigma_{r r}$ must be uniform along radial lines. Since this stress component is zero on the lateral surface, it must be zero everywhere. By the first equation of system (13), this implies that

$$
a=\frac{\alpha-3}{\alpha+6} c
$$

The final value of the plastic multiplier $\eta$ then follows from equations (12) and (15):

$$
\eta=\frac{1}{2 \sigma_{0}} \sqrt{\frac{b^{2} r^{2}}{3}+\frac{6 c^{2}}{\alpha+6}}
$$


and the final expressions of the stress components follow from combination of equations (13) and this result:

$$
\left\{\begin{array}{l}
\sigma_{r r}=\sigma_{\theta \theta}=0 \\
\sigma_{z z}=\sigma_{0} \frac{6 c}{(\alpha+6) \sqrt{\frac{b^{2} r^{2}}{3}+\frac{6 c^{2}}{\alpha+6}}} \\
\sigma_{\theta z}=\sigma_{0} \frac{b r}{3 \sqrt{\frac{b^{2} r^{2}}{3}+\frac{6 c^{2}}{\alpha+6}}} .
\end{array}\right.
$$

It has already been said that the case of a Green material has not been considered in earlier works. However, in the special case of a von Mises material $(\alpha=0)$, expressions (17) coincide, with appropriate changes of notation, with (i) those provided by Hill (1950), Drucker (1967) and Lubliner (2008) in the rigid-ideal-plastic case; (ii) those given by Gaydon (1952) and Chakrabarty (1987) in the elastic-ideal-plastic case, in the limit of very large elastic moduli.

Some final comments on the method of derivation are noteworthy:

- In the approach adopted, the radial velocity $v_{r}$ is assumed to be a linear function of $r$; the ultimate consequence of this postulated property is that both stress components $\sigma_{r r}$ and $\sigma_{\theta \theta}$ are uniformly zero within the cylinder. The sequence of logical steps leading to this conclusion is as follows: (i) the linearity of $v_{r}$ in $r$ implies that $\dot{\epsilon}_{r r}-\dot{\epsilon}_{\theta \theta}=0$ and therefore, by the flow rule, that $s_{r r}-s_{\theta \theta}=0$; (ii) the radial equilibrium equation then implies that $d \sigma_{r r} / d r=0$ so that $\sigma_{r r}$ must be a constant, which can only be zero since the lateral boundary is not loaded; (iii) the equality of $s_{r r}$ and $s_{\theta \theta}$ implies the equality of $\sigma_{r r}$ and $\sigma_{\theta \theta}$, so that $\sigma_{\theta \theta}$ must also be uniformly zero.

- One could equally well, conversely, start from the reasonable assumption that $\sigma_{r r}$ and $\sigma_{\theta \theta}$ are uniformly zero, and end up with the conclusion that $v_{r}$ must be a linear function of $r$. The reasoning would be as follows: (i) since $\sigma_{r r}=\sigma_{\theta \theta}=0, \sigma_{r r}-\sigma_{\theta \theta}=s_{r r}-s_{\theta \theta}=0$; (ii) combined with the flow rule, this implies that $\dot{\epsilon}_{r r}-\dot{\epsilon}_{\theta \theta}=0$; (ii) thus necessarily $d v_{r} / d r-v_{r} / r=0$ which implies that $v_{r}=a r$ for some constant $a$.

\section{Parametric form of the overall yield locus}

Varying the pairs of kinematic parameters $(b, c)$ in equations $(17)$, one obtains all possible solution stress fields $\left\{\sigma_{z z}, \sigma_{\theta z}\right\}$, and therefore all pairs of load parameters $(F, T)$ inducing unrestrained plastic flow of the cylinder. Now it is clear from equations (4) and (17) that starting from a pair $(b, c)$ generating the fields $\left\{\sigma_{z z}, \sigma_{\theta z}\right\}$ and the pair $(F, T)$, and considering the 4 pairs $( \pm b, \pm c)$, one obtains the 4 fields $\left\{ \pm \sigma_{z z}, \pm \sigma_{\theta z}\right\}$ and therefore the 4 pairs $( \pm F, \pm T)$. This means that the overall yield locus, in the $(F, T)$-plane, is symmetric with respect to both coordinate axes plus the origin. We shall therefore focus hereafter on the study of the sole upper-right quadrant of this locus corresponding to positive values of $\sigma_{z z}$ and $\sigma_{\theta z}$, that is by equations (17) to positive parameters $b$ and $c$.

Combination of equations (4) and (17) yields the following integral expressions of the 
tension force and torsion torque:

$$
F=\sigma_{0} \int_{0}^{R} \frac{6 c}{(\alpha+6) \sqrt{\frac{b^{2} r^{2}}{3}+\frac{6 c^{2}}{\alpha+6}}} .2 \pi r d r \quad ; \quad T=\sigma_{0} \int_{0}^{R} \frac{b r}{3 \sqrt{\frac{b^{2} r^{2}}{3}+\frac{6 c^{2}}{\alpha+6}}} .2 \pi r^{2} d r
$$

The calculation of these integrals is elementary using the change of variable $u \equiv \frac{\alpha+6}{18} \frac{b^{2}}{c^{2}} r^{2}$. Accounting for the positiveness of $b$ and $c$, one gets

$$
\left\{\begin{array}{l}
\bar{F} \equiv \frac{F}{\sqrt{\frac{6}{\alpha+6}} \pi R^{2} \sigma_{0}}=\frac{2}{\xi^{2}}\left(\sqrt{\xi^{2}+1}-1\right) \\
\bar{T} \equiv \frac{T}{\frac{2 \pi R^{3} \sigma_{0}}{3 \sqrt{3}}}=\frac{1}{\xi^{3}}\left[\left(\xi^{2}-2\right) \sqrt{\xi^{2}+1}+2\right]
\end{array} \quad \text { where } \quad \xi \equiv \sqrt{\frac{\alpha+6}{18}} \frac{b}{c} R .\right.
$$

In the definition of the "reduced", dimensionless tension force $\bar{F}$ and torsion torque $\bar{T}$ here, the denominators $\sqrt{\frac{6}{\alpha+6}} \pi R^{2} \sigma_{0}$ and $\frac{2 \pi R^{3} \sigma_{0}}{3 \sqrt{3}}$ represent the values of the tension force and torsion torque inducing unrestrained plastic flow in pure tension $\left(\xi \rightarrow 0^{+}\right)$and pure torsion $(\xi \rightarrow+\infty)$, respectively.

Equations (19) represent parametric equations of the overall yield locus, in terms of the parameter $\xi$ connected to the ratio $\dot{\phi} / \dot{\delta}$ of the torsional angular rate $\dot{\phi}$ to the axial extension rate $\dot{\delta}$ (see equations (3)). They are, quite remarkably, independent of the coefficient $\alpha$ characterizing the dependence of Green's criterion (5) upon the mean stress: the influence of this coefficient occurs only through the value of the limit-load in pure tension, not through a modification of the functional form of the overall criterion which is the same as for von Mises's criterion $(\alpha=0)$.

Again, equations (19) are identical, with appropriate changes of notation, to (i) those provided by Drucker (1967) and Lubliner (2008) in the rigid-ideal-plastic case; (ii) those given by Gaydon (1952) and Chakrabarty (1987) in the elastic-ideal-plastic case, in the limit of very large elastic moduli. Note however that previous authors considered only the case of von Mises's criterion, without noticing the more general applicability of their results.

\section{Explicit expression of the overall yield locus}

We shall now show how the parameter $\xi$ may be eliminated between the expressions (19) of the reduced tension force $\bar{F}$ and torsion torque $\bar{T}$, so as to get an explicit equation of the overall yield locus.

Let us set

$$
\xi \equiv \sinh \zeta \quad\left(\text { so that } \sqrt{\xi^{2}+1}=\cosh \zeta\right) .
$$


The expressions (19) of $\bar{F}$ and $\bar{T}$ then become

$$
\left\{\begin{array}{l}
\bar{F}=\frac{2}{\sinh ^{2} \zeta}(\cosh \zeta-1)=\frac{2}{\cosh \zeta+1} \\
\bar{T}=\frac{1}{\sinh ^{3} \zeta}\left[\left(\sinh ^{2} \zeta-2\right) \cosh \zeta+2\right]=\frac{1}{\sinh ^{3} \zeta}\left(\cosh ^{3} \zeta-3 \cosh \zeta+2\right) .
\end{array}\right.
$$

Use of the identity $x^{3}-3 x+2=(x-1)^{2}(x+2)$ in the second equation of system $(21)$ yields

$$
\begin{aligned}
\bar{T}^{2} & =\frac{1}{\sinh ^{6} \zeta}(\cosh \zeta-1)^{4}(\cosh \zeta+2)^{2}=\frac{(\cosh \zeta-1)^{4}(\cosh \zeta+2)^{2}}{\left(\cosh ^{2} \zeta-1\right)^{3}} \\
& =\frac{(\cosh \zeta-1)(\cosh \zeta+2)^{2}}{(\cosh \zeta+1)^{3}} .
\end{aligned}
$$

Writing $\cosh \zeta$ as $\cosh \zeta+1-1$ in the numerator and $(\cosh \zeta+1)^{3}$ as $(\cosh \zeta+1)(\cosh \zeta+1)^{2}$ in the denominator, one transforms this expression into

$$
\begin{aligned}
\bar{T}^{2} & =\frac{\cosh \zeta+1-2}{\cosh \zeta+1} \frac{(\cosh \zeta+1+1)^{2}}{(\cosh \zeta+1)^{2}}=\left(1-\frac{2}{\cosh \zeta+1}\right)\left(1+\frac{1}{\cosh \zeta+1}\right)^{2} \\
& =(1-\bar{F})\left(1+\frac{\bar{F}}{2}\right)^{2}
\end{aligned}
$$

where the first equation of system (21) has been used. It follows that the equation of the quadrant of the overall yield locus considered, $[\bar{F}>0, \bar{T}>0]$, reads

$$
\Phi(\bar{F}, \bar{T}) \equiv(\bar{F}-1)\left(\frac{\bar{F}}{2}+1\right)^{2}+\bar{T}^{2}=0 .
$$

It is easy to find the equation of the full yield locus from there, using the symmetry properties mentioned in Section 4:

$$
\Phi(\bar{F}, \bar{T}) \equiv(|\bar{F}|-1)\left(\frac{|\bar{F}|}{2}+1\right)^{2}+\bar{T}^{2}=0 .
$$

The function $\Phi(\bar{F}, \bar{T})$ defined here is easily checked to be convex, which entails convexity of the region defined by the inequality $\Phi(\bar{F}, \bar{T}) \leq 0$, that is of the domain of sustainable loads. (Such a property is no surprise since it in fact holds for all limit-analysis problems, being a straightforward consequence of the definition of sustainable loads as those for which there exists a stress field which is both statically admissible with them and plastically admissible).

Equation (23) for the overall yield locus is identical to, though of more general applicability than, that derived by Lubliner (2008) in the special case of the von Mises criterion. It combines the three features mentioned in the Introduction: be non-trivial (as a result of the non-uniformity of the solution stress field), exact and explicit. Examples of solutions of limit-analysis problems exhibiting such a combination are scarce in the literature. 
The red curve in Figure 2 (where $\bar{F}$ and $\bar{T}$ are noted "Fbar" and "Tbar") shows the quadrant of the yield locus defined by equation (22). (The comparison with the case of a thin tube, also illustrated in the figure, will be commented in Subsection 7.1 below).

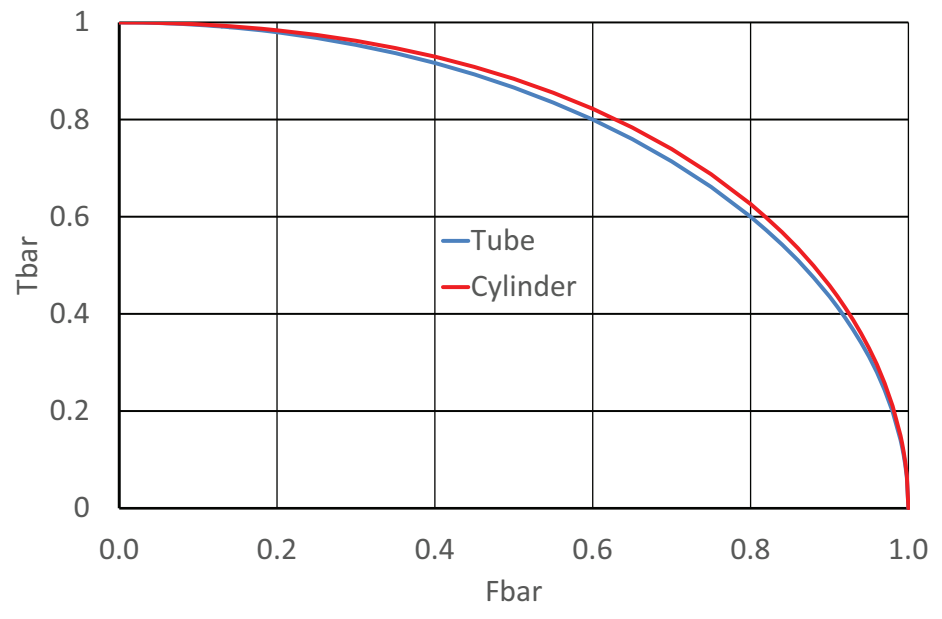

Fig. 2. Overall yield loci of a thin tube and a circular cylinder

\section{Overall flow rule}

A classical result of limit-analysis, stated with maximum clarity by Mandel (1966), asserts the following: if the loads considered depend linearly upon $N$ load parameters $Q_{1}, \ldots, Q_{N}$, then the rates of the conjugate kinematic parameters $q_{1}, \ldots, q_{N}$ at a limit-load obey some "overall normality rule":

$$
\dot{q}_{i}=H \frac{\partial \Phi}{\partial Q_{i}}\left(Q_{1}, \ldots, Q_{N}\right) \quad, \quad H \geq 0
$$

where $\Phi\left(Q_{1}, \ldots, Q_{N}\right)$ is the "overall yield function" and $H$ the "overall plastic multiplier". (This result is a consequence of the following result of Hill $(1951)$ : if $\left(Q_{1}, \ldots, Q_{N}\right)$ denotes a limit-load, $\left(\dot{q}_{1}, \ldots, \dot{q}_{N}\right)$ the corresponding vector of rates of conjugate kinematic parameters, and $\left(Q_{1}^{*}, \ldots, Q_{N}^{*}\right)$ a sustainable load, then necessarily $\sum_{i=1}^{N}\left(Q_{i}-Q_{i}^{*}\right) \dot{q}_{i} \geq 0$.)

In the case of the cylinder in combined tension and torsion, a natural definition of the two load parameters is

$$
Q_{1} \equiv \bar{F} \quad ; \quad Q_{2} \equiv \bar{T}
$$

where $\bar{F}$ and $\bar{T}$ are the reduced tension force and torsion torque defined by equations (19). To identify the conjugate parameters $q_{1}, q_{2}$, one must evaluate the virtual power $\mathcal{P}_{e}^{*}$ of external forces in an arbitrary motion characterized by the virtual rates $\dot{\delta}^{*}, \dot{\phi}^{*}$ of the axial extension $\delta$ (relative axial displacement of the upper and lower faces) and torsional angle $\phi$ (relative angle of rotation of the upper and lower faces about the $O z$ axis). This 
power is given by

$$
\begin{aligned}
\mathcal{P}_{e}^{*} & =\int_{\text {Upper face }}\left(\sigma_{z z} \dot{\delta}^{*}+\sigma_{\theta z} \dot{\phi}^{*} r\right) d S=\int_{0}^{R}\left[\sigma_{z z}(r) \dot{\delta}^{*}+\sigma_{\theta z}(r) \dot{\phi}^{*} r\right] 2 \pi r d r \\
& =F \dot{\delta}^{*}+T \dot{\phi}^{*}=\sqrt{\frac{6}{\alpha+6}} \pi R^{2} \sigma_{0} \bar{F} \dot{\delta}^{*}+\frac{2 \pi R^{3} \sigma_{0}}{3 \sqrt{3}} \bar{T} \dot{\phi}^{*}
\end{aligned}
$$

where equations (4) and (19) have been used. It then follows from the property characterizing conjugate kinematic parameters, $\mathcal{P}_{e}^{*}=Q_{1} \dot{q}_{1}{ }^{*}+\ldots+Q_{N} \dot{q}_{N}{ }^{*}$ in every possible virtual motion, that

$$
q_{1} \equiv \sqrt{\frac{6}{\alpha+6}} \pi R^{2} \sigma_{0} \delta \quad ; \quad q_{2} \equiv \frac{2 \pi R^{3} \sigma_{0}}{3 \sqrt{3}} \phi
$$

Focussing again on the upper-right quadrant of the overall yield locus $[\bar{F}>0, \bar{T}>0]$, equations $(24,25,26)$ yield, account being taken of the expression (22) of the overall yield function $\Phi$ :

$$
\frac{\dot{q}_{2}}{\dot{q}_{1}}=\frac{\sqrt{2(\alpha+6)}}{9} \frac{R \dot{\phi}}{\dot{\delta}}=\frac{\partial \Phi / \partial Q_{2}}{\partial \Phi / \partial Q_{1}}\left(Q_{1}, Q_{2}\right)=\frac{\partial \Phi / \partial \bar{T}}{\partial \Phi / \partial \bar{F}}(\bar{F}, \bar{T})=\frac{4}{3} \frac{\bar{T}}{\bar{F}\left(\frac{\bar{F}}{2}+1\right)}
$$

or equivalently, using again equation (22) to express $\bar{T}$ as a function of $\bar{F}$ :

$$
\frac{\sqrt{2(\alpha+6)}}{9} \frac{R \dot{\phi}}{\dot{\delta}}=\frac{4}{3} \frac{\sqrt{1-\bar{F}}}{\bar{F}} \Rightarrow \frac{R \dot{\phi}}{\dot{\delta}}=6 \sqrt{\frac{2}{\alpha+6}} \frac{\sqrt{1-\bar{F}}}{\bar{F}} .
$$

Equation (27) provides the "direction of overall plastic flow" as a function of the sole reduced tension force. It may also be obtained directly - thus providing a check on the correctness of the overall flow rule (24) resulting from the overall normality property - by solving the first equation of system (19) with respect to $\xi$ :

$$
\frac{\bar{F}}{2} \xi^{2}+1=\sqrt{\xi^{2}+1} \Rightarrow \frac{\bar{F}^{2}}{4} \xi^{4}+\bar{F} \xi^{2}+1=\xi^{2}+1 \Rightarrow \frac{\bar{F}^{2}}{4} \xi^{2}=1-\bar{F} \Rightarrow \xi=2 \frac{\sqrt{1-\bar{F}}}{\bar{F}} ;
$$

this is equivalent to equation (27) since $\xi=\sqrt{\frac{\alpha+6}{18}} \frac{b}{c} R=\sqrt{\frac{\alpha+6}{18}} \frac{R \dot{\phi}}{\dot{\delta}}$ by equation (3) and the definition (19) of $\xi$.

The red curve in Figure 3 shows the quantity (noted "Normal to yield locus") $\frac{\partial \Phi / \partial \bar{T}}{\partial \Phi / \partial \bar{F}}$ (= $\frac{\sqrt{2(\alpha+6)}}{9} \frac{R \dot{\phi}}{\dot{\delta}}$ ) defined by equation (27). (Again, the comparison with the case of a thin tube, also illustrated in the figure, will be commented in Subsection 7.1 below). 


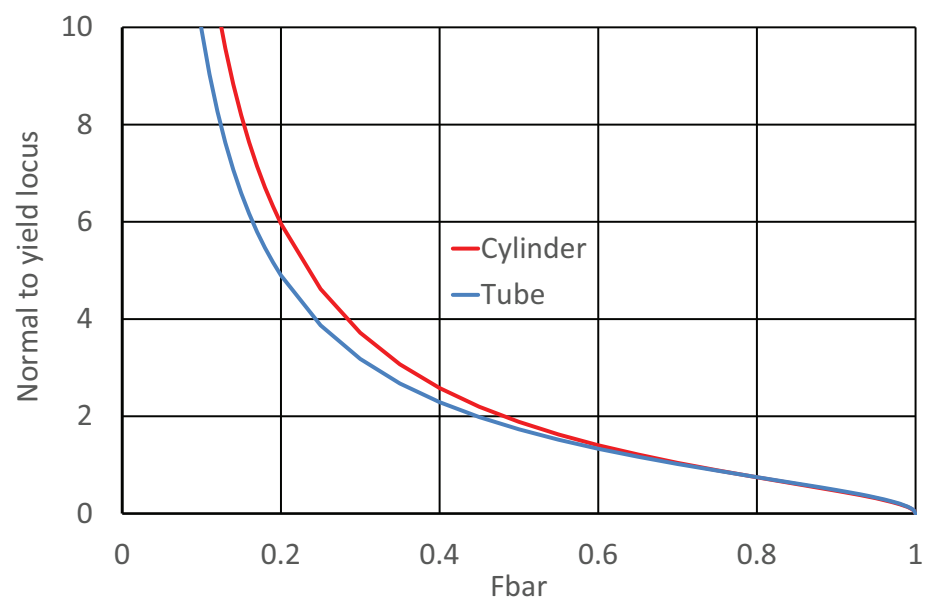

Fig. 3. Normals to the overall yield loci of a thin tube and a circular cylinder

\section{Discussion}

\subsection{Comparison with the case of a thin tube}

It is interesting to compare the results derived above for a cylinder to those for a thin tube subjected to the same loading. For the latter geometry the nonzero components of the stress field are the same; but their uniformity, combined with the quadratic character of the Green criterion, implies that the overall yield locus can only be a circle of equation $\bar{F}^{2}+\bar{T}^{2}=1$, provided that the quantities $\bar{F}$ and $\bar{T}$ are re-defined as the ratios of the tension force $F$ and torsion torque $T$ over their maximum possible values for the new geometrical configuration considered.

This second yield locus is represented in blue in Figure 2. The two yield loci are very close, in spite of the differences in the geometries considered and the non-uniformity of the solution stress field in the cylinder versus the homogeneity of that in the thin tube. The fact that the overall yield locus for a cylinder is almost circular was noted for the first time by Drucker (1967), but in the sole case of the von Mises criterion). As a complement, Figure 4 displays the relative difference between the two reduced torsion torques versus the reduced tension force. This relative difference never exceeds $6 \%$.

However the difference between the normals is more conspicuous, as can be seen in Figure 3 which also represents, in blue, the quantity $\frac{\partial \Phi / \partial \bar{T}}{\partial \Phi / \partial F}$ for a thin tube. Again, as a complement, Figure 5 displays the relative difference between the two normals versus the reduced tension force. This relative difference is observed to reach a value of about $25 \%$ for small values of $\bar{F}$, which is significant and should be observable experimentally.

\subsection{Practical significance of the overall yield function and flow rule}

The theory of limit-analysis is based on the assumption of ideal plasticity. But few materials may safely be considered as ideal-, or nearly ideal-plastic. It thus necessary to discuss 


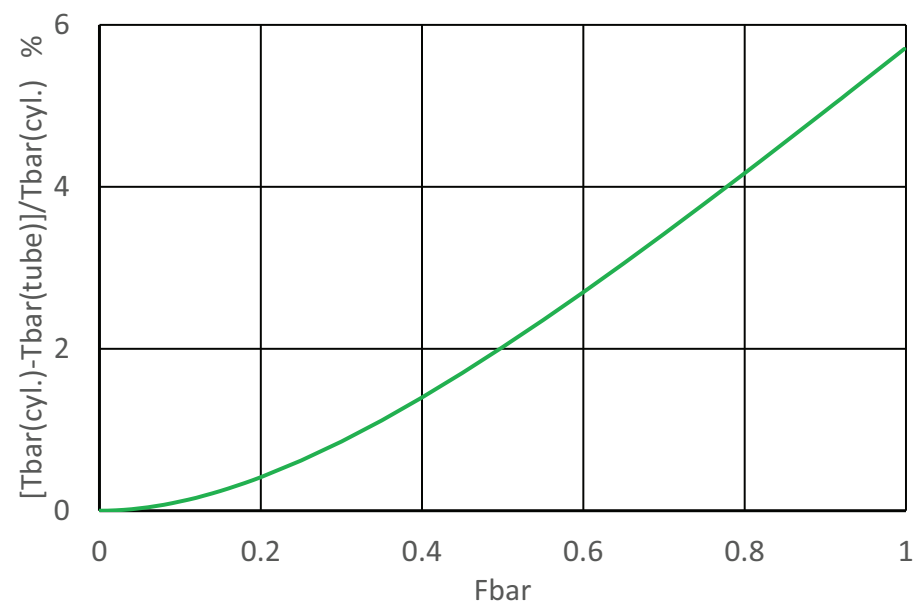

Fig. 4. Relative gap between the overall yield loci of a thin tube and a circular cylinder

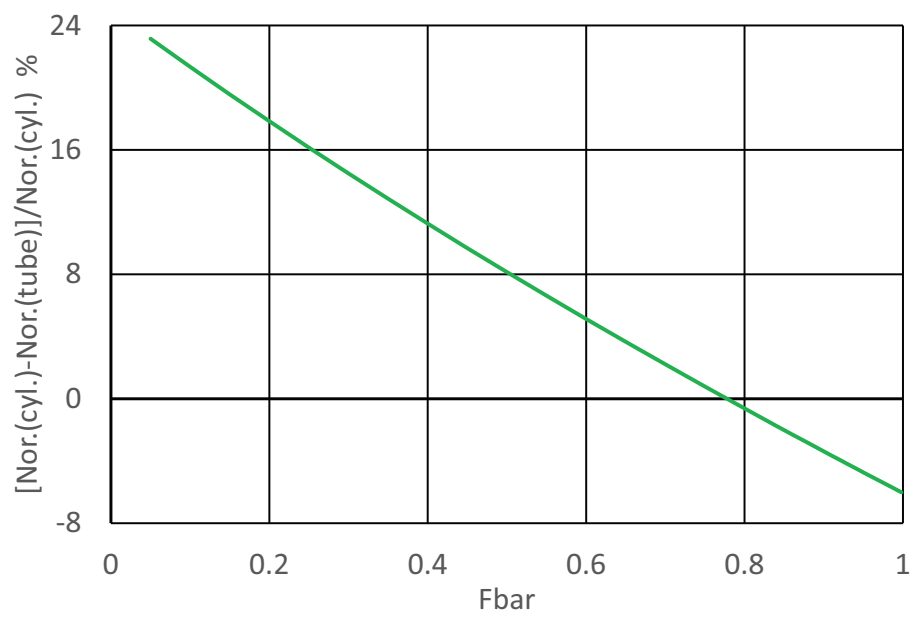

Fig. 5. Relative gap between the normals to the overall yield loci of a thin tube and a circular cylinder

the conditions under which the limit-analysis solution developed above may be applied to the majority of materials, which exhibit more or less significant strain hardening.

The idea is, of course, to apply this solution to the prediction of the initial yield point of the cylinder, defined as the lowest load ensuring plastic behaviour everywhere in the structure. The problem then arises: because of the presence of elasticity, except in the special case of pure tension, plasticity does not start at all points simultaneously, but progresses gradually inwards from the lateral outer surface toward the axis. Thus, when plasticity reaches the axis, points located on the surface have already experienced some strain hardening. This hardening must not be "too large" for the limit-analysis solution based on the hypothesis of ideal plasticity to be applicable. This means that hardening must not be "too important" at the beginning of the stress-strain curve; the ideal case being that of a metal exhibiting a significant Piobert-Lüders plateau.

To make the analysis more quantitative, consider a cylinder made of some elastic-plastic material and subjected to some purely torsional load. (This is the "most unfavourable" load in the sense that it generates the longest possible period of partial plasticity of the structure, the plastic zone filling the cylinder only asymptotically, in the limit of very 
large torsion angles). To get just a rough order of magnitude of the ratio of the strains on the outer surface and near the axis, consider the yield stress $k \equiv \sigma_{0} / \sqrt{3}$ in pure shear as a constant. Denote $R_{e}(\leq R)$ the radius of the central (cylindrical) elastic zone, when the value of the torsion torque is $T$. The stress component $\sigma_{\theta z}$ varies linearly with $r$ (like the deformation $\epsilon_{\theta z}$ ) within the elastic region $0 \leq r<R_{e}$ and is uniform and equal to $k$ within the plastic region $R_{e} \leq r \leq R$. It then follows from equation (4) that

$$
T=\int_{0}^{R_{e}} k \frac{r}{R_{e}} \cdot 2 \pi r^{2} d r+\int_{R_{e}}^{R} k \cdot 2 \pi r^{2} d r=T^{L}\left[1-\frac{1}{4}\left(\frac{R_{e}}{R}\right)^{3}\right]
$$

where $T^{L} \equiv \frac{2 \pi}{3} k R^{3}=\frac{2 \pi R^{3} \sigma_{0}}{3 \sqrt{3}}$ denotes the asymptotic value of $T$, obtained in the limit $R_{e} \rightarrow 0$.

Now assume that $T$ has almost reached this asymptotic value $T^{L}$; say $T=0.99 T^{L}$. Then, by what precedes, $\frac{1}{4}\left(\frac{R_{e}}{R}\right)^{3}=0.01$, implying that $R_{e} / R \simeq 0.34$. Now the deformation $\epsilon_{\theta z}$ is proportional to $r$; thus it is then only $1 / 0.34 \simeq 2.9$ times higher on the outer surface $(r=R)$ than on the interface between the elastic and plastic regions $\left(r=R_{e}\right)$. This means that when the structure may be considered as "sufficiently plastic" for the "fully plastic" solution to be applicable, the strain on the outer surface is only roughly 3 times larger than that strain corresponding to onset of plastic yield.

Thus the limit-analysis solution based on the neglect of strain hardening is reasonable provided that the variation of the yield stress is negligible between the initial yield point and that corresponding to a strain 3 times larger. In particular it is acceptable if the material exhibits a Piobert-Lüders plateau extending beyond the triple of its initial strain.

\subsection{Possible application to the measurement of the parameter $\alpha$}

Application of Green (1972)'s criterion (5) to structural problems involving highly porous plastic materials of course requires experimental determination of the parameter $\alpha$ characterizing the influence of the mean stress in this criterion. We finally discuss how the results derived above could be used for this purpose. Cylindrical specimens and tension/torsion loads represent the simplest, most practical choice for experiments aimed at such a determination. ${ }^{2}$

Since real materials are hardenable, the limit-analysis solution derived above is not applicable after a significant amount of global deformation. But it is probably reasonable to assume the parameter $\alpha$ to be a constant independent of deformation, since its value is mainly determined by the volume fraction, shape and distribution of voids, rather than by the global deformation. With such a hypothesis, one may determine $\alpha$ from observations made at the beginning of global plastic flow of the cylinder, provided of course that the

$\overline{2}$ One may a priori envisage to perform even simpler experiments in pure tension; in theory $\alpha$ could then be determined by comparing the axial and transverse strains. But accurate measurement of the transverse strain would be difficult. 
conditions for validity of the limit-analysis solution detailed in Subsection 7.2 above are met.

The simplest idea would of course be to compare the experimental values of the limit-loads of the cylinder in pure tension $\left(\sqrt{\frac{6}{\alpha+6}} \pi R^{2} \sigma_{0}\right)$ and in pure torsion $\left(\frac{2 \pi R^{3} \sigma_{0}}{3 \sqrt{3}}\right)$. The ratio of these limit-loads is independent of $\sigma_{0}$ but depends on $\alpha$, so that its value would directly yield the value of the latter parameter, circumventing the need for determination of the former.

However measuring limit-loads is often a difficult task in the absence of a sharply defined yield point. It may therefore be preferable to use the overall plastic flow rule in the form of equation (27). Measurement of the ratio $\frac{R \dot{\phi}}{\dot{\delta}}$ would be easy and provide the value of the quantity $6 \sqrt{\frac{2}{\alpha+6}} \frac{\sqrt{1-\bar{F}}}{F}$. Measurement of $\bar{F}$ would then yield the value of $\alpha$.

One foreseeable drawback, however, is that measurement of $\bar{F}$ would require measuring not only the axial force $F$ - an easy task - but also its maximum possible value in pure tension, which brings us back to the same problem as before of inaccuracy of measurements of yield points. One way of circumventing this difficulty would be to avoid direct measurement of the limit-load in pure tension, considering instead this limit-load as an extra unknown which could be determined from the flow rule together with the parameter $\alpha$ using a least-squares method; this would just require performing experiments for several (at least two) experiments corresponding to different values of the ratio of the tension torque to the axial force.

Of course, this procedure can only be hoped to work for sufficiently large values of $\alpha$, that is highly porous materials. Indeed for small values of $\alpha, \alpha+6$ differs little from 6 so that one cannot expect an accurate determination of $\alpha$ from a measurement of this sum.

\section{Acknowledgement}

Jean-Baptiste Leblond acknowledges financial support by the Institut Universitaire de France under grant éOTP L10K018.

\section{Compliance with ethical standards}

The authors declare they have no conflict of interest.

\section{References}

Avitzur B. and Pan J.Z. (1985). Cylinder under combined axial and torsion load, Int. J. Mach. Tool Des. Res., 25, 269-284. 
de Buhan P. (2007). Plasticité et Calcul à la Rupture, Presses des Ponts et Chaussées.

Chakrabarty J. (1987). Theory of Plasticity, McGraw-Hill.

Corapcioglu Y. and Uz T. (1985). Constitutive equations for plastic deformation of porous materials, Powder Technol., 21, 269-274.

Drucker D.C. (1967). Introduction to Mechanics of Deformable Solids, McGraw-Hill.

Drucker D.C., Prager W. and Greenberg M.J. (1952). Extended limit-analysis theorems for continuous media, Quart. Appl. Math., 9, 381-389.

Fritzen F., Forest S., Kondo D. and Boehlke T. (2013). Computational homogenization of porous materials of Green type, Comput. Mech., 9, 121-134.

Gaydon F.A. (1952). On the combined torsion and tension of a partly plastic circular cylinder, Quart. J. Mech. Appl. Math., 5, 29-41.

Green R.J. (1972). A plasticity theory for porous solids, Int. J. Mech. Sci., 14, 215-224.

Gurson A.L. (1977). Continuum theory of ductile rupture by void nucleation and growth: Part I - Yield criteria and flow rules for porous ductile media, ASME J. Engng. Mater. Technol., 99, 2-15.

Hill R. (1950). The Mathematical Theory of Plasticity, Oxford University Press.

Hill R. (1951). On the state of stress in a plastic-rigid body at the yield point, Phil. Mag., 42, 868-875.

Kuhn H.A. and Downey C.L. (1971). Deformation characteristics and plasticity theory of sintered powder materials, Int. J. Powder Metall., 7, 15-25.

Lubliner J. (2008). Plasticity Theory, Dover Publications.

Mandel J. (1966). Cours de Mécanique des Milieux Continus, Gauthier-Villars.

Nadah J., Bignonnet F., Davy C.A., Skoczylas F., Troadec D. and Bakowski S. (2013). Microstructure and poro-mechanical performance of Haubourdin chalk, Int. J. Rock Mech. Mining Sci., 58, 149-165.

Oyane M., Omura M., Tabata T. and Hisatsune T. (1989). An upper bound approach on yield surfaces of porous materials, Ingenieur-Archiv, 59, 267-273.

Prager W. and Hodge P.G. (1951). Theory of Perfectly Plastic Solids, Wiley and Sons.

Save M.A., Massonet C.E. and de Saxcé G. (1997). Plastic Limit Analysis of Plates, Shells and Disks, North-Holland.

Salençon J. (1983). Calcul à la Rupture et Analyse-Limite, Presses de l'Ecole Nationale des Ponts et Chaussées.

Shao J.F. and Henry J.P. (1991). Developpement of an elastoplastic model for porous rock, Int. J. Plast., 7, 1-13.

Shen W.Q., Shao J.F., Dormieux L. and Kondo D. (2012). Approximate criteria for ductile porous materials having a Green type matrix: Application to double porous media, Comput. Mater. Sci., 62, 189-194.

Shima S. and Oyane M. (1976). Plasticity for porous metals, Int. J. Mech. Sci., 18, 286291. 